\title{
Effective treatment of a platinum-resistant cutaneous squamous cell carcinoma case by EGFR pathway inhibition
}

\author{
CARLO CAPALBO $^{1,2^{*}}$, FRANCESCA BELARDINILLI $^{1 *}$, MARCO FILETTI $^{2}$, CLAUDIA PARISI $^{2}$, \\ MARIALAURA PETRONI ${ }^{3}$, VALERIA COLICCHIA ${ }^{1}$, ALESSANDRA TESSITORE ${ }^{2}$, \\ MATTEO SANTONI $^{5}$, ANNA COPPA ${ }^{6}$, GIUSEPPE GIANNINI ${ }^{1,7^{*}}$ and PAOLO MARCHETTI ${ }^{2 *}$ \\ ${ }^{1}$ Department of Molecular Medicine, Sapienza University of Rome, I-00161 Rome; \\ ${ }^{2}$ Medical Oncology Sant'Andrea Hospital, I-00189 Rome; ${ }^{3}$ Center for Life Nano Science@ Sapienza, \\ Istituto Italiano di Tecnologia, I-00161 Rome; ${ }^{4}$ Department of Biotechnological and Applied Clinical Sciences, \\ University of L'Aquila, I-67100 L'Aquila; ${ }^{5}$ Oncology Unit, Macerata Hospital, I-62100 Macerata; ${ }^{6}$ Department of \\ Experimental Medicine, Sapienza University of Rome; ${ }^{7}$ Istituto Pasteur-Fondazione Cenci Bolognetti, I-00161 Rome, Italy
}

Received November 10, 2017; Accepted March 30, 2018

DOI: $10.3892 / \mathrm{mco} .2018 .1634$

\begin{abstract}
Cutaneous squamous cell carcinoma (cSCC) is the second most common type of non-melanoma skin cancer. Platinum-based regimens have been an integral part of palliative care for patients with locally advanced or metastatic disease. There is no evidence of efficacy for later lines of chemotherapy and no targeted therapy has been introduced as 'standard of care'. Here we report on the case of an elderly cSCC patient, resistant to conventional therapy, however successfully treated with anti-epidermal growth factor receptor (EGFR) agent (Cetuximab) in addition to a daily dose of Curcumin phospholipid. The patient responded to treatment and experienced no recurrence for 11 months with only minor skin-related toxicity. To our knowledge, this is the first report of clinical evidence that an anti EGFR targeted therapy with a daily oral dose of Curcumin phospholipid is well tolerated and results in a highly effective disease control in a heavily pretreated cSCC patient.
\end{abstract}

\section{Introduction}

Cutaneus Squamous Cell Carcinoma (cSCC) is a common malignancy in elderly, representing nearly $25 \%$ of non-melanoma skin cancers. Its incidence is continuously rising due to aging population and increased ultraviolet exposure (1).

Correspondence to: Dr Carlo Capalbo or Dr Giuseppe Giannini, Department of Molecular Medicine, Sapienza University of Rome, Laurenziano 9, I-00161 Rome, Italy

E-mail: carlo.capalbo@uniroma1.it

E-mail: giuseppe.giannini@uniroma1.it

*Contributed equally

Key words: cSCC, targeted therapy, cetuximab, curcumin, next-generation sequencing, Ion Torrent PGM, mutations, EGFR pathway, FGFR3
Platinum-based combination therapies demonstrated high efficacy in the locally advanced or metastatic settings. However, they are not always able to guarantee durable responses (2), highlighting the need to assess new therapeutic options, especially for the recurrent disease no more amenable to surgery.

So far, no targeted therapy has been introduced as 'standard of care' for cSCC. However, multiple studies have reported deregulation of the EGFR-signaling cascades (RAS-MAPK and PI3K-AKT-mTOR axes) and/or other pathways (i.e., Notch, p53, CDKN2A) (3,4). A recent comprehensive genomic profiling of 315 cancer genes in $122 \mathrm{cSCC}$ cases showed that $88 \%$ harbored at least one clinically relevant mutation, with an average of 2.5 actionable genomic alterations per patient (5). Thus, conventional and unconventional deregulated pathways eventually amenable for therapeutic intervention are being found also in cSCC. In example, anti-EGFR monoclonal antibodies have been used in platinum-resistant advanced cSCC patients with clinical benefit and improved toxicity profiles (6).

Curcumin is a dietary polyphenol derived from the root of the plant Curcuma Longa, which has been shown to possess anti-inflammatory and anti-cancer activities both in vitro and in vivo $(7,8)$. In vitro, it causes cell growth inhibition and/or apoptosis in multiple cancer cell models (9) and has been reported to inhibit several cancer-related pathways such as PI3K-AKT-mTOR and EGFR axes (10). In particular, Curcumin demonstrated to reduce the invasion and adhesive abilities of cSCC A431 cells by the inhibition of Signal Transducer and Activator of Transcription 3 (STAT3) activation (11). Furthermore, Phillips et al reported that Curcumin was able to significantly reduce cSCC tumor progression in vivo by inhibiting S6 phosphorylation and, as a consequence, the mTOR pathway (12).

Here we report the first description of an elderly cSCC patient, resistant to conventional treatments, but successfully responding to an anti-EGFR pathway inibition with a 'chemo-free' combination of Cetuximab with a daily oral dose of Curcumin phospholipid supplement. 


\section{Data collection methods}

DNA extraction. DNA was extracted from Formalin-Fixed and Paraffin-Embedded (FFPE) tumor tissue, obtained by skin lesion biopsy. Xylene was added once and ethanol was added twice to remove all paraffin from the tissue sample. The DNA was extracted using QIAamp DNA FFPE Tissue kit (Qiagen $\mathrm{GmbH}$, Hilden, Germany) according to the manufacturer's instructions (13). Eluted DNA was quantified with Qubit 2.0 Fluorometer (Thermo fisher).

IT-PGM sequencing and variant calling. Approximately $10 \mathrm{ng}$ of DNA was required to construct the barcoded and adaptor-ligated library using the Ion AmpliSeq Library Kit 2.0 (Thermo fisher). The sample was analysed using the Ion AmpliSeq Colon and Lung Cancer Research Panel V2 containing a single primer pool to amplify hotspots and targeted regions of 22 cancer genes frequently mutated in CRCs and NSCLCs (https://www.ampliseq.com). Templated spheres were prepared using $100 \mathrm{pM}$ of the library using the Ion One Touch 2.0 machine. Template-positive spheres were loaded into Ion chip 314 and sequenced by IT-PGM machine (Thermo fisher). Sequencing data were finally analysed with Coverage Analysis and Variant Caller plugins available within the Ion Torrent Suite software. Variants with a quality $<30$ were filtered out. Sequence reads were finally visualized with IGV tool using HG19 as reference genome to direct inspection of mutations.

A
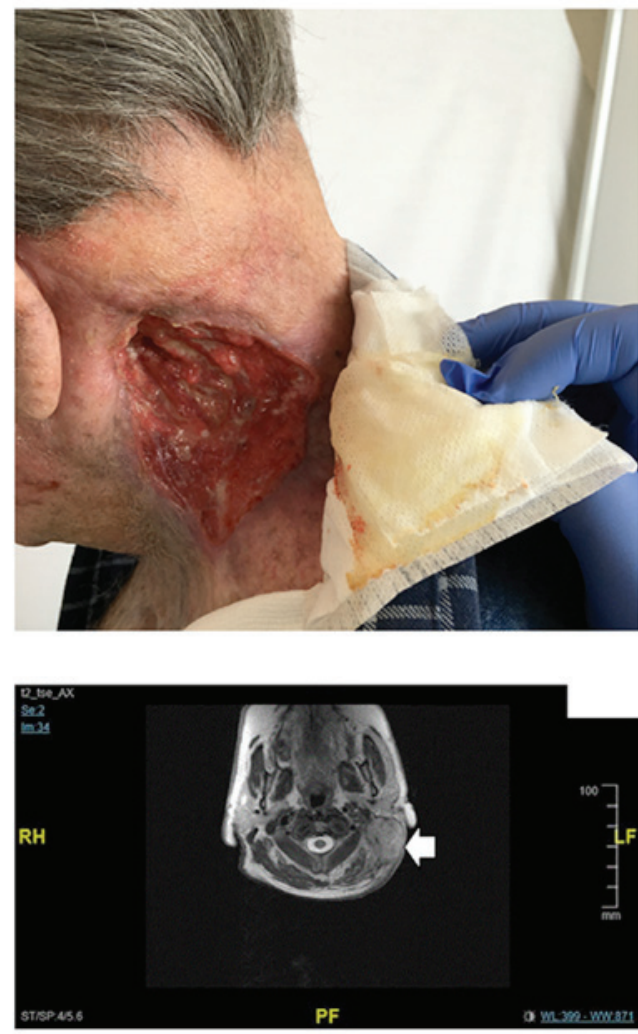

\section{Case report}

An 83 years old Caucasian male patient was admitted in our oncology department on March 2016 for recurrent cSCC of the supraclavicular region. The patient had been previously treated with a platinum-based chemotherapy regimen (Carboplatin AUC4-5-Fluorouracil, 750 mg day 1-5; q21-3 cycles) with neoadjuvant intent. After an early locoregional disease progression, he had debulking surgery followed by locoregional radiotherapy. More specifically, the patient underwent radical ipsilateral neck dissection with resection of the sternocleidomastoid muscle, the spinal accessory nerve, the whole collarbone and the internal jugular vein, required to remove the vast (m.d. $15 \times 8 \mathrm{~cm}$ ) lesion infiltrating the underlying tissue. The pathology report confirmed the diagnosis of poorly differentiated cSCC obtained at the time of first biopsy and revealed bone infiltration.

After 9 months, a head and neck MRI revealed a locoregional disease progression characterized by a $6.4 \times 3.6 \mathrm{~cm}$ nuchal lesion and a $10.5 \times 4.8 \mathrm{~cm}$ lesion in the supraclavicular region having a cranium-caudal extension of $15 \mathrm{~cm}$. The tumor had also infiltrated the left paravertebral muscle, the left parotid gland and the lax cellular tissue of the supraclavicular region. An increase in cervical, submandibular and nuchal lymph nodes dimension was also detectable. Moreover, the supraclavicular $10 \mathrm{~cm}$ lesion appeared ulcerated at the medical examination (Fig. 1A).

Given the extent of disease and its lack of response to previous treatments, a new systemic therapy appeared

B
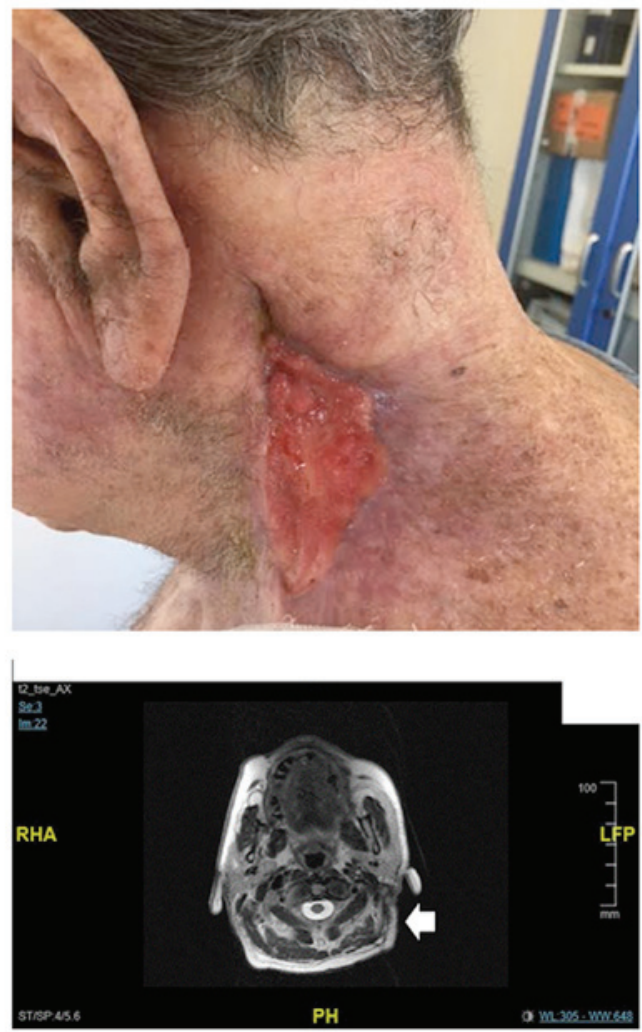

Figure 1. Lesion and MRI of the patient before and after cetuximab-curcumin treatment for recurrent cSCC of the supraclavicular region. Tumor masse (arrow) can be seen in the nuchal and supraclavicular regions of the patient before initiation of cetuximab-curcumin treatment. Moreover, the supraclavicular $10 \mathrm{~cm}$ lesion appeared ulcerated at the medical examination (A). The masse (arrow) became significantly reduced in size on MRI obtained 12 weeks after treatment (B), indicating good response to combination treatment. 
Table I. Genetic variants found in the cSCC patient by IT-PGM sequencing.

\begin{tabular}{|c|c|c|c|c|c|}
\hline Gene $^{\mathrm{a}}$ & Mutation $^{\mathrm{b}}$ & $\mathrm{AF}^{\mathrm{c}}(\%)$ & Relevance $^{\mathrm{d}}$ & Prediction test $\mathrm{t}^{\mathrm{j}}$ & (Refs.) \\
\hline \multirow[t]{2}{*}{ FGFR3 } & c.1138G>A p.Gly380Arg (COSM24842) & 28.7 & Pathogenic $c^{e, f}$ & & $(23,24)$ \\
\hline & c. $1953 \mathrm{G}>$ A p.Thr651=(rs7688609) & 100.0 & Benign $^{e, g}$ & & \\
\hline \multirow[t]{2}{*}{ EGFR } & c. $1498+22$ A >T (rs 1558544) & 100.0 & $\mathrm{NC}^{\mathrm{g}, \mathrm{i}}$ & NNSPLICE: Unchanged & \\
\hline & c. $2361 \mathrm{G}>$ A p $. \mathrm{G} \ln 787=(\mathrm{rs} 1050171)$ & 100.0 & Benign $^{\mathrm{e}, \mathrm{g}}$ & & \\
\hline MET & c.534C >T p.Ser178Ser (rs35775721) & 67.2 & Benign $^{g}$ & & \\
\hline \multirow[t]{4}{*}{ MAP2K1 } & c. $174 \mathrm{G}>\mathrm{C}$ p.Gln58His & 15.8 & Likely pathogenic ${ }^{\mathrm{i}}$ & PROVEAN: Deleterius; & \\
\hline & & & & SIFT: Damaging; & \\
\hline & & & & PolyPhen: Possibly damaging; & \\
\hline & & & & $\begin{array}{l}\text { CRAVAT: High pathogenicity } \\
\text { cancer driver impact }\end{array}$ & \\
\hline \multirow[t]{5}{*}{ TP53 } & c.743G>A p. Arg248Gln (COSM10662) & 21.1 & Pathogenic $c^{\mathrm{e}, \mathrm{fh}}$ & & $(25-27)$ \\
\hline & c.836_861del27 & 31.1 & Likely pathogenic ${ }^{\mathrm{i}}$ & PROVEAN: Deleterius & \\
\hline & p.Gly279_Asn288delinsAsp & & & $\begin{array}{l}\text { CRAVAT: High pathogenicity } \\
\text { cancer driver impact }\end{array}$ & \\
\hline & $\begin{array}{l}\text { c.215 C>G p.Pro72Arg, } \\
\text { (rs 1042522, COSM250061) }\end{array}$ & 60.2 & $\begin{array}{l}\text { Uncertain } \\
\text { significance }^{e, f, i}\end{array}$ & & $(30)$ \\
\hline & c. $1-46 \mathrm{C}>\mathrm{T}$ & 21.2 & NA & NNSPLICE: Unchanged & \\
\hline
\end{tabular}

${ }^{a}$ The following 22 genes were analyzed: DDR2, NRAS, ALK, ERBB4, CTNNB1, PIK3CA, FBXW7, FGFR3, BRAF, EGFR, MET, FGFR1, NOTCH1, FGFR2, PTEN, KRAS, AKT1, MAP2K1, ERBB2, TP53, SMAD4, STK11. 'Sequence variant nomenclature according to HGVS recommendations (http://varnomen.hgvs.org/); (COSMIC and/or dbSNP ID numbers). ${ }^{\mathrm{A}} \mathrm{AF}$ : Variant allele frequency in the tumor sample. ${ }^{\mathrm{d} B i o l o g i c a l}$ impact of the variant according to: ${ }^{\mathrm{e}} \mathrm{Clin} \operatorname{Var}$ (https://www.ncbi.nlm.nih.gov/clinvar/); ${ }^{\mathrm{f}} \mathrm{COSMIC} \mathrm{(http://cancer.sanger.ac.uk/cosmic);}$

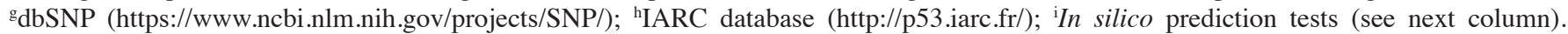
In silico prediction on the biological impact of the variant according to multiple software (PolyPhen-2, http://genetics.bwh.harvard.edu/pph2/; SIFT, http://sift.jcvi.org/; PROVEAN, http://provean.jcvi.org/index.php; CRAVAT, https://www.cravat.us/CRAVAT/; NNSPLICE, http://www. fruitfly.org/seq_tools/splice.html.

necessary for the patient. In order to consider alternative treatment opportunities dictated by tumor biology, we evaluated the mutational profile of the tumor at the time of the latest progression. DNA was extracted from FFPE tumor tissue, obtained by skin lesion biopsy. This was sequenced on an Ion Torrent PGM platform by the Ion AmpliSeq Colon and Lung research Panel V2. The tumor sample showed no mutation in most relevant EGFR signal transducers. In contrast, it harbored pathogenic mutations in the FGFR3 and TP53 genes and a likely pathogenic mutation in the MAP2K1 gene (Table I).

The lack of other established therapeutic alternatives, the positive results obtained by a recent phase II Study (14) employing Cetuximab and the absence of mutations on major components of the EGFR signal transduction pathway met the ethical constraints of a tailored therapy, and prompted us to propose an off-label Cetuximab-based combination treatment.

Based on its known anti-inflammatory and anti-tumor properties and in consideration of its ability to inhibit cancer-related pathways, in this particular case we added a daily supplementation of oral Curcumin phospholipid (Meriva, $500 \mathrm{mg}$ orally once daily) to the conventional Cetuximab schedule $(400 \mathrm{mg} / \mathrm{mq}$, followed by subsequent weekly doses of $250 \mathrm{mg} / \mathrm{mq}$ ). The protocol was approved by the institutional review board (Ospedale Sant'Andrea, Sapienza University of Rome) on March 2016, and the patient provided written informed consent. Already after 4 weeks of this treatment, a significant clinical benefit was reported by the patient. In particular, the lesion's bleeding stopped and a $5 \mathrm{~cm}$ reduction in size was reported at the medical examination. After 12 weeks, a head and neck MRI confirmed the clinical response and showed an $\sim 50 \%$ reduction of the lesions. The infiltration of the left parotid gland was no longer detectable and a dramatic regression in cervical, submandibular and nuchal lymph nodes was evidenced (Fig. 1B). Thus, the patient responded to treatment and experienced no recurrence for 11 months. After 12 weeks, no major therapy-related toxicity had been observed. On the contrary, treatment regimen had been well tolerated and patient complained only for minor skin-related toxicity (maximum grade 2 according to CTCAE version 4.0).

\section{Discussion}

In recent years, given the limited efficacy of standard-of-care chemotherapy and radiotherapy for patients with locally advanced or systemic disease, several investigators have begun to study the genomic background of cSCC looking for novel actionable targets. In particular, the widespread employment of NGS techniques allowed to identify a large number of potentially actionable driver genes $(15,16)$. As previously recognized for other tumors of epithelial origin, the 
RAS-RAF-MEK-ERK and the PI3K/AKT branches acting downstream of RTKs such as EGFR are very frequently mutated/deregulated also in cSCC (17). Nonetheless, no targeted therapy options have entered the routine and wide use for patients with cSCC, yet $(18,19)$. EGFR inhibitors should, in principle, be considered a valid therapeutic option also for cSCC, since EGFR seems to be overexpressed in a high proportion of primary tumors of this type, which acquire a metastatic phenotype (20). The absence of mutations in most relevant RTK-signal transducers revealed by our molecular profiling allowed us to offer the patient a therapeutic intervention based on Cetuximab (chimeric mouse-human anti-EGFR $\mathrm{IgG1}$ monoclonal antibody). Initially approved for the treatment of advanced colorectal cancer, and subsequently employed in advanced or platinum-refractory Head and Neck Squamous Cell Carcinoma (HNSCC), Cetuximab monotherapy has also proved its efficacy and low toxicity profile in advanced and metastatic cSCC, as described in several case reports or case series $(21,22)$.

In particular, in a recent prospective phase II trial carried out by Maubec et al, comprehensive of 36 patients, a $67 \%$ rate of 6-weeks disease control supported the use of single agent Cetuximab as first-line treatment for unresectable cSCC (14).

Nonetheless, the activation of alternative intracellular networks involved in the maintenance of the malignant phenotype could also limit the benefit of anti-EGFR treatment.

In our specific case, genetic profiling of the tumor tissue showed pathogenic mutations in FGFR3, TP53 and MAP2K1 genes. The p.Gly380Arg FGFR3 mutation has been reported in patients affected with achondroplasia, the most common form of human dwarfism (23), and in cancer patients (24). It causes an increased and ligand-independent phosphorylation of FGFR3 leading to constitutive activation of the downstream pathway, largely accounted for by MAPK and PI3K/AKT activity. Two different TP53 mutations coexisted in our cSCC case: The p.Arg248Gln is a known gain of function p53 mutation (25-27), while the p.Gly279_Asn288delinsAsp is a previously undescribed in-frame deletion which is predicted to be highly pathogenic. The low allelic frequency of both TP53 mutations suggests that both alleles of this gene might be destroyed in the tumor tissue. The MAP2K1 p.Gln58His mutation is also previously undescribed, but multiple prediction tools indicate it is most likely pathogenic (Table I). In principle, both the FGFR3 and the MAP2K1 mutations could have impaired the effect of anti-EGFR treatment, in this patient.

Interestingly, Curcumin is endowed with potent antinflammatory and cancer chemopreventive and therapeutic properties. Although the precise molecular mechanism of Curcumin action is far from being completely understood, it regulates the expression of several genes involved in cytokines production, cellular proliferation and cell survival (28), part of which are known TP53 targets.

Recent studies have pointed out that pharmacological doses of Curcumin can inhibit EGFR pathway in different squamous malignancies (8). Moreover, it has also been shown to enhance the inhibitory effect generated by drugs directly targeting EGFR, while also improving their toxicity profile (9). Preclinical evidences further indicated its ability to overcome anti-EGFR therapy resistance (29). All of these observations provided the rational for its use in combination with Cetuximab in the reported case. While we are aware that the description of a single cSCC case does not allow us to draw major conclusion on the possibility that Curcumin phospholipid oral supplement might potentiate the efficacy of the anti-EGFR treatment, it is important to notice that this strategy was well tolerated and resulted in a highly effective control of the disease in a heavily pretreated cSCC patient.

Based on our preliminary observation, we believe that the benefit of a combined anti-EGFR/Curcumin treatment on molecularly stratified cSCC patients should be further addressed with a specific clinical trial.

\section{Acknowledgements}

Not applicable.

\section{Funding}

The present study was supported by grants from: Associazione Italiana per la Ricerca sul Cancro (grant no. IG17734), Ministry of University and Research, PRIN projects and Istituto Pasteur-Fondazione Cenci Bolognetti to GG.

\section{Availability of data and materials}

All data generated or analyzed during this study are included in this published article.

\section{Authors' contributions}

$\mathrm{CC}, \mathrm{CP}, \mathrm{GG}, \mathrm{MF}$ and PM were involved in patient recruitment. $\mathrm{FB}, \mathrm{MP}, \mathrm{VC}, \mathrm{AT}$ and $\mathrm{AC}$ were involved in sample preparation and sequencing. $\mathrm{CC}, \mathrm{FB}, \mathrm{MS}, \mathrm{PM}, \mathrm{GG}$ were involved in data analysis. CC, FB, MF, CP, MP, VC, AT, MS, AC, GG, and PM were involved in manuscript writing and editing.

\section{Ethics approval and consent to participate}

Not applicable.

\section{Consent for publication}

Written informed consent was obtained from the patient for the publication of this case.

\section{Competing interests}

The authors declare that they have no competing interests.

\section{References}

1. Green AC and Olsen CM: Cutaneous squamous cell carcinoma: An epidemiological review. Br J Dermatol 177: 373-381, 2017.

2. Behan JW, Sutton A and Wysong A: Management of skin cancer in the high-risk patient. Curr Treat Options Oncol 17: 60, 2016.

3. Swick AD, Prabakaran PJ, Miller MC, Javaid AM, Fisher MM, Sampene E, Ong IM, Hu R, Iida M, Nickel KP, et al: Cotargeting mTORC and EGFR signaling as a therapeutic strategy in HNSCC. Mol Cancer Ther 16: 1257-1268, 2017.

4. Iglesias-Bartolome R, Martin D and Gutkind JS: Exploiting the head and neck cancer oncogenome: Widespread PI3K-mTOR pathway alterations and novel molecular targets. Cancer Discov 3: 722-725, 2013. 
5. Al-Rohil RN, Tarasen AJ, Carlson JA, Wang K, Johnson A, Yelensky R, Lipson D, Elvin JA, Vergilio JA, Ali SM, et al: Evaluation of 122 advanced-stage cutaneous squamous cell carcinomas by comprehensive genomic profiling opens the door for new routes to targeted therapies. Cancer 122: 249-257, 2016.

6. Suen JK, Bressler L, Shord SS, Warso M and Villano JL: Cutaneous squamous cell carcinoma responding serially to single-agent cetuximab. Anticancer Drugs 18: 827-829, 2007.

7. Yang X, Li Z, Wang N, Li L, Song L, He T, Sun L, Wang Z, Wu Q, Luo N, et al: Curcumin-encapsulated polymeric micelles suppress the development of colon cancer in vitro and in vivo. Sci Rep 5: 10322, 2015.

8. Starok M, Preira P, Vayssade M, Haupt K, Salomé L and Rossi C: EGFR inhibition by curcumin in cancer cells: A dual mode of action. Biomacromolecules 16: 1634-1642, 2015.

9. Wada K, Lee JY, Hung HY, Shi Q, Lin L, Zhao Y, Goto M, Yang PC, Kuo SC, Chen HW and Lee KH: Novel curcumin analogs to overcome EGFR-TKI lung adenocarcinoma drug resistance and reduce EGFR-TKI-induced GI adverse effects. Bioorg Med Chem 23: 1507-1514, 2015.

10. Kasi PD, Tamilselvam R, Skalicka-Woźniak K, Nabavi SF, Daglia M, Bishayee A, Pazoki-Toroudi $\mathrm{H}$ and Nabavi SM: Molecular targets of curcumin for cancer therapy: An updated review. Tumour Biol 37: 13017-13028, 2016.

11. Wu J, Lu WY and Cui LL: Inhibitory effect of curcumin on invasion of skin squamous cell carcinoma A431 cells. Asian Pac J Cancer Prev 16: 2813-2818, 2015.

12. Phillips JM, Clark C, Herman-Ferdinandez L, Moore-Medlin T, Rong X, Gill JR, Clifford JL, Abreo F and Nathan CO: Curcumin inhibits skin squamous cell carcinoma tumor growth in vivo. Otolaryngol Head Neck Surg 145: 58-63, 2011.

13. Giannini G, Capalbo C, Ottini L, Buffone A, De Marchis L, Margaria E, Vitolo D, Ricevuto E, Rinaldi C, Zani M, et al: Clinical classification of BRCA1 DNA missense variants: H1686Q is a novel pathogenic mutation occurring in the ontogenetically invariant THV motif of the N-terminal BRCT domain J Clin Oncol 26: 4212-4214; author reply, 4214-4215, 2008.

14. Maubec E, Petrow P, Scheer-Senyarich I, Duvillard P, Lacroix L, Gelly J, Certain A, Duval X, Crickx B, Buffard V, et al: Phase Il study of cetuximab as first-line single-drug therapy in patients with unresectable squamous cell carcinoma of the skin. J Clin Oncol 29: 3419-3426, 2011

15. Belardinilli F, Capalbo C, Buffone A, Petroni M, Colicchia V, Ferraro S, Zani M, Nicolussi A, D'Inzeo S, Coppa A, et al: Validation of the Ion Torrent PGM sequencing for the prospective routine molecular diagnostic of colorectal cancer. Clin Biochem 48: 908-910, 2015.

16. Capalbo C, Marchetti P, Coppa A, Calogero A, Anastasi E, Buffone A, Belardinilli F, Gulino M, Frati P, Catalano C, et al: Vemurafenib and panitumumab combination tailored therapy in BRAF-mutated metastatic colorectal cancer: A case report Cancer Biol Ther 15: 826-831, 2014.

17. Toll A, Salgado R, Yébenes M, Martín-Ezquerra G, Gilaberte M, Baró T, Solé F, Alameda F, Espinet B and Pujol RM: Epidermal growth factor receptor gene numerical aberrations are frequent events in actinic keratoses and invasive cutaneous squamous cell carcinomas. Exp Dermatol 19: $151-153,2010$.
18. Schwaederle M, Elkin SK, Tomson BN, Carter JL and Kurzrock R: Squamousness: Next-generation sequencing reveals shared molecular features across squamous tumor types. Cell Cycle 14: 2355-2361, 2015.

19. Pickering CR, Zhou JH, Lee JJ, Drummond JA, Peng SA, Saade RE, Tsai KY, Curry JL, Tetzlaff MT, Lai SY, et al: Mutational landscape of aggressive cutaneous squamous cell carcinoma. Clin Cancer Res 20: 6582-6592, 2014.

20. Shimizu T, Izumi H, Oga A, Furumoto H, Murakami T, Ofuji R, Muto M and Sasaki K: Epidermal growth factor receptor overexpression and genetic aberrations in metastatic squamous-cell carcinoma of the skin. Dermatology 202: 203-206, 2001.

21. Wollina U: Cetuximab in non-melanoma skin cancer. Expert Opin Biol Ther 12: 949-956, 2012.

22. Seber S, Gonultas A, Ozturk O and Yetisyigit T: Recurrent squamous cell carcinoma of the skin treated successfully with single agent cetuximab therapy. Onco Targets Ther 9: 945-948, 2016.

23. Takagi M, Kouwaki M, Kawase K, Shinohara H, Hasegawa Y, Yamada T, Fujiwara I, Sawai H, Nishimura G and Hasegawa T: A novel mutation Ser344Cys in FGFR3 causes achondroplasia with severe platyspondyly. Am J Med Genet A 167A: 2851-2854, 2015.

24. L'Hôte CG and Knowles MA: Cell responses to FGFR3 signalling: Growth, differentiation and apoptosis. Exp Cell Res 304: 417-431, 2005.

25. Shtraizent N, Matsui H, Polotskaia A and Bargonetti J: Hot spot mutation in TP53 (R248Q) causes oncogenic gain-of-function phenotypes in a breast cancer cell line derived from an African American patient. Int J Environ Res Public Health 13: ijerph13010022, 2015.

26. Muller PA and Vousden KH: Mutant p53 in cancer: New functions and therapeutic opportunities. Cancer Cell 25: 304-317, 2014.

27. Xu J, Wang J, Hu Y, Qian J, Xu B, Chen H, Zou W and Fang JY: Unequal prognostic potentials of p53 gain-of-function mutations in human cancers associate with drug-metabolizing activity. Cell Death Dis 5: e1108, 2014.

28. Jobin C, Bradham CA, Russo MP, Juma B, Narula AS, Brenner DA and Sartor RB: Curcumin blocks cytokine-mediated NF-kappa $\mathrm{B}$ activation and proinflammatory gene expression by inhibiting inhibitory factor I-kappa B kinase activity. J Immunol 163 : 3474-3483, 1999.

29. Li S, Liu Z, Zhu F, Fan X, Wu X, Zhao H and Jiang L: Curcumin lowers erlotinib resistance in non-small cell lung carcinoma cells with mutated EGF receptor. Oncol Res 21: 137-144, 2013.

30. Olivier M, Hollstein M and Hainaut P: TP53 mutations in human cancers: Origins, consequences, and clinical use. Cold Spring Harb Perspect Biol 2: a001008, 2010. 